\title{
Leiomyoma and leiomyoma cellulare of the fallopian tube: review of the literature and case reports
}

\author{
Dobrosława L. Sikora-Szczęśniak \\ Department of Obstetrics and Gynecology, Specialist Hospital in Radom, Poland
}

\begin{abstract}
Introduction: Leiomyoma of the fallopian tube is extremely rare, and its version - leiomyoma cellulare (LC) of the fallopian tube is absolutely unique. Aim of the study was to review literature reports on leiomyomas of the fallopian tubes, and to present cases of leiomyoma and LC of the fallopian tubes in the patients operated on in our ward.

Material and methods: There were fewer than 100 cases of leiomyomas of the fallopian tubes discussed in the literature up to 1993. Case 1. Leiomyoma of the left fallopian tube was detected postoperatively in a 68-yearold patient, G.K., on histopathological examination after laparoscopic total hysterectomy with bilateral adnexa. Case 2. A 56-year-old patient, K.T., with LC of the fallopian tube was qualified for laparoscopy. At operation, the procedure was converted to microlaparotomy due to the tumor size. The adnexa on the right side with the tumor of the fallopian tube were excised, and the left fallopian tube was excised, too. Histopathological microscopy found leiomyoma cellulare partim epithelioides.

Results: In the presented cases, the extent of operation was connected with the clinical picture, and in the case of LC of the right fallopian tube, with intraoperative histopathological findings. In both cases the postoperative course was uneventful.

Conclusions: Diagnosis of leiomyoma and LC of the fallopian tube, like in the other organs of the female genital tract, is possible only due to results of histopathological microscopy.
\end{abstract}

Key words: leiomyoma of the fallopian tube, leiomyoma cellulare of the fallopian tube.

\section{Introduction}

Leiomyoma cellulare (LC) is the most common benign tumor of the female genital tract, including the uterine body. It is a benign non-epithelial, mesenchymal monoclonal tumor of smooth muscle cells and a framework of fibrous connective tissue [1, 2]. Uterine LC occurs with $5 \%$ frequency [3].

These tumors develop as genetically abnormal clones of cells originating from one precursor cell where the original mutation took place. Multiple leiomyomas do not belong to the same clone; each develops irrespectively of the others [4]. Their more common occurrence in first-degree relatives suggests genetic background of the disease [5].

Recently, more and more attention has been paid to the role of genes (tuberous sclerosis complex gene - TSC) in the etiology of malignant and benign tumors. Lost function of those genes manifested as a decreased level of TSC gene product, i.e. tuberin was observed in $50 \%$ of cases of uterine leiomyomas, which suggests genetic etiology of the disease [6-8].
Clinically LC appears as solid nodules of large smooth muscle cells and their nuclei without atypia, of low mitotic activity up to 5 division figures/10 HPF, with scarce connective tissue elements. It is often referred to as cellular leiomyoma [9-11].

On postoperative macro- and microscopic examination, the mass of removed LC tumors and uteruses was considerably larger compared to leiomyomas [12].

Leiomyoma of the fallopian tube originates from the myosalpinx or the cells of the blood vessels supplying the fallopian tube. In most cases it is located in the isthmus, rarely in the ampulla, but it is also found as myoma nascens. Similarly to uterine leiomyomata, the leiomyoma of the fallopian tube may be located submucosally, intramurally or subserosally. When large enough or in statu nascendi they tend to twist or degenerate $[13,14]$.

The paper aims at reviewing literature reports on leiomyoma of the fallopian tube and its variant, leiomyoma cellulare. Moreover, it presents two case reports of the patients operated on in our ward. Microscopic histological examination found leiomyoma parvum of 
the left fallopian tube (case 1), and leiomyoma cellulare partim epithelioides of the right fallopian tube (case 2).

\section{Material and methods}

The paper reviews accessible research articles published in the last decades on the cases of leiomyoma of the fallopian tube, and a case of LC of the fallopian tube. The analysis focused on:

- preoperative diagnostic methods and preoperative diagnosis,

- patient's age, body mass index (BMI), and obstetric history,

- methods of treatment and extent of surgery,

- results of surgical treatment.

Moreover, we present two case reports of the patients operated on in our ward: one patient had leiomyoma of the left fallopian tube, and the other LC of the right fallopian tube; both diagnoses were made on microscopic histopathological examination.

\section{Results}

Leiomyoma of the fallopian tube is rare and often incidentally detected at surgery or autopsy [15]. In the majority of cases preoperative diagnosis is sporadic as the disease course is asymptomatic, and those tumors are singular, small size, and unilaterally located nodules $[15,16]$.

When the mass of a leiomyoma located in the fallopian tube is larger, the so called "effect of mass" can cause additional symptoms from the urinary bladder, ureters or the alimentary tract [17]. Moreover, torsion, or degeneration is unlikely to be found until surgery and histopathological examination [14].

Imaging techniques, such as transvaginal ultrasonography (USG) Doppler, computed tomography (CT) and magnetic resonance imaging (MRI) are useful for preoperative diagnostics as they can detect a leiomyoma mass in the fallopian tube, the location other than the uterus. However, the final diagnosis is made intraoperatively by results of histological examination [16].

The age of patients with leiomyoma of the fallopian tube, whose cases are presented in the papers, ranged from 27 to 44 years [13-20]. The mean age of the patients in the group with uterine leiomyomas and leiomyomas and endometriosis was 49.1 years [3].

The age of the patients operated on in our ward, of 68 and 56 years, was higher than the mean age of 45.6 years noted in the group of patients with uterine LC [3].

Ozkan et al. presented a case of a 70-year-old patient with LC of the fallopian tube [21].

The risk factors include nulliparity, advanced age, obesity, and family history of leiomyomas [17].

The operative technique and extent of the operation depended upon the patient's age, size of the lesion in the fallopian tube and the result of intraoperative histopathological examination. Laparoscopy was used as a diagnostic technique to localize the mass and for intraoperative histopathological examination of the sample.

The large mass of the diseased fallopian tube and coexisting diseases affecting the internal genital organs were decisive for the extent of the operation, sometimes conversion of laparoscopy into laparotomy was necessary [13].

\section{Case reports of the patients operated on in our ward \\ Cose 1}

The patient, G.K., 68 years, was admitted for uterine leiomyomas and recurrent uterine bleeding; menopause at 55 , four natural deliveries.

Preoperative diagnosis and patient's medical history and examination found obesity (class I, body mass $67 \mathrm{~kg}, \mathrm{BMI}=32.80$ ). Additionally, the patient was on medication for hypertension and coronary heart disease.

Surgical operation: hysterectomy with bilateral adnexa by laparoscopic approach was performed on 3 December 2002.

Findings of postoperative histological examination of the material:

- the uterine body - Endometrium senile cysticum. Endometriosis diffusa. Leiomyomata intramuralia et subserosa unius cellulare corporis uter,

- the uterine cervix - Erosio glandularis partim epidermisata cum inflammatione. Ovula Nabothi,

- left appendages - Cystis simplex ovarii atrophici. Leiomyoma parvum oviducti,

- the right appendages - Ovarium. Oviductus.

The patient was discharged on the $6^{\text {th }}$ day following the operation.

\section{Case 2}

The patient, K.T., 53 years, admitted for a tumor in the right appendages on 18 March 2015.

History findings: regular menstruation from the age of 14 to 53; five natural deliveries. Preoperative diagnosis, patient's history and physical examination revealed obesity (class II, body mass $95 \mathrm{~kg}, \mathrm{BMI}=37.11$ ); additionally the patient was on medication for hypertension, type 2 diabetes, and schizophrenia.

Laboratory tests were done and pharmacological treatment of concomitant diseases continued as consulted by specialists.

Laboratory results found temporary elevated blood glucose 173 mg/dl. Complete blood count (CBC), electrolytes, kidney parameters and blood coagulation parameters were within normal range. CA125 23.16 U/ml within the normal range. 
Gynecological examination findings: vaginal section of the uterine cervix normal, muco-serous fluid scarce. The uterus and appendages difficult to examine due to the patient's obesity.

Ultrasound scan (Fig. 1): the uterus body not enlarged, size of $5 \times 3 \times 3 \mathrm{~cm}$. Endometrium $5.4 \mathrm{~mm}$ thick. On the right side, a cyst $55 \times 50 \mathrm{~mm}$ in diameter filled with thick fluid, the appendages on the left side normal.

Preoperative diagnosis of the cyst of the right fallopian tube was made, and the patient was qualified for laparoscopy on 24 March 2015.

Surgical operation - in the course of laparoscopy, the operator decided on conversion to minilaparotomy because of the tumor size of $80 \times 60 \times 55 \mathrm{~mm}$. The right appendages with the tumor of the right fallopian tube were excised, and left salpingectomy was performed.

Intraoperative examination found a benign lesion of the right fallopian tube - tumor of $80 \times 60 \times 55 \mathrm{~mm}$, lobular, grayish, of smooth surface, growing at the terminal part the fallopian tube; the fallopian tube unwidened, along it numerous spotty whitish cyst-like nodules filled with whitish fluid.

Histopathological examination under microscope (No 996010-7/15) found:

- tumor of the right fallopian tube - Leiomyoma cellulare partim epithelioides, of atypical nuclei, mitotic index 0 ,

- right fallopian tube-Oviductus,

- right ovary-Ovarium,

- left fallopian tube (No. 995981/15): fallopian tube $70 \mathrm{~cm}$ - long, $5 \mathrm{~mm}$ in diameter, with singular minute cysts on the surface - Oviductus et cystis serosa paraoviducti.

The patient was discharged from the ward on the $4^{\text {th }}$ day following the operation. The postoperative course was uneventful in all the cases described in the literature, as well as in the patients operated on in our ward.

\section{Discussion}

There are only few reports on leiomyoma of the fallopian tube in the literature as its incidence is defined as extremely rare $[13,16,18,20]$.

In their review of the literature, Mroueh et al. quoted fewer than 100 cases of leiomyomas of the fallopian tubes [22]. Since then several researchers have discussed those cases, including one of LC of the fallopian tube $[13-21,23,24]$. It is difficult to evaluate the frequency and risk of $L C$ of the fallopian tube as the number of cases is very small [17].

The cases of LC variant located in the extrauterine region are really incidental. There are only two cases described in the Polish literature [3, 25]. Among the leiomyomas and LC cases, LC in the extrauterine region and LC in the uterine cervix accounted for $0.34 \%$ and $6.25 \%$, respectively [3].

As seen in the literature review, the presented case of LC of the fallopian tube is the second one reported [21].

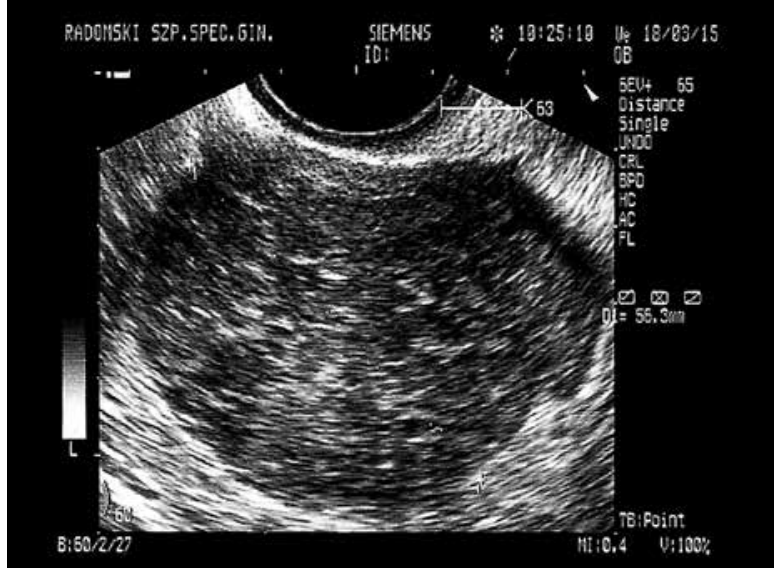

Fig. 1. Ultrasound view leyomyoma cellulare of fallopian tube

In the discussed cases of leiomyoma of the fallopian tube, the patients' age was $27,32,34,35,39,41,44$, and 44 years [respectively 13-20].

The mean age in the group of the patients with leiomyomas and leiomyomas with endometriosis was $49.1 \mathrm{yrs}$ [3]. Thus, the age of the patients operated on in our ward, of 68 and 56 years, was higher compared to the mean age of 45.6 years [3].

The patient with LC of the fallopian tube, whose case is presented by Ozkan et al., was 70 [21].

Yang et al. state that leiomyomas of the fallopian tube are generally small nodules located unilaterally [16]. In the case presented above, the tumor mass was considerably large compared to the size of the fallopian tube itself. Ozkan et al. found LC of the fallopian tube of $130 \times 90 \times 70 \mathrm{~mm}[21]$.

Takongmo et al. and Cisse et al. described detected leiomyomas of the fallopian tube as "giant" $[13,17]$.

Moreover, torsion of leiomyomas of the fallopian tube and degeneration were also reported [13-15, 19, 24].

There are also cases of uterine pregnancies and a case of ectopic pregnancy (in the fallopian tube) in the patients with leiomyoma of the fallopian tube [17, $22,23]$. In the case of giant tumor in the right fallopian tube, the pregnancy was spontaneously aborted [17].

The operative method and technique were commensurate with the size of the diseased fallopian tube, concomitant problems affecting internal genital organs, and the result of intraoperative histopathological examination.

When the fallopian tube affected by LC ruptured and bleeding to the peritoneal cavity occurred, emergency laparotomy was performed [21].

Preoperative diagnostics emphasize the importance of imaging techniques (USG Doppler, CT, MRI) for the diagnosis of leiomyoma of the fallopian tube [16]. If suspected, the diagnosis is confirmed by laparoscopy and results of intraoperative histopathological examination. That is what exactly happened in the presented 
case when it was necessary to convert laparoscopy into minilaparotomy. Similar decisions were taken in other presented cases, too [16, 19].

Sometimes preoperative imaging is not enough to define the nature of a lesion, however it is generally very useful to locate the change.

In the above-presented cases, preoperative diagnosis considered mesenteric tumor, abdominal tumor, tumor of the pelvis minor, bleeding to the peritoneal cavity, torsion of the subserosal uterine leiomyoma [13, $14,17,19,21]$.

In the case of our patient, the initial diagnosis was ovarian cyst.

Concomitant diseases of the genital organs that are the reason for operation can pose other difficulties in detecting leiomyoma of the fallopian tube by imaging techniques, especially if the lesion is small. That happened in case 1 presented above.

It supports incidental detection of the majority of cases at surgery or autopsy [14-16, 18].

Genetic research by Lobel et al. found that larger leiomyoma uteri tumors more frequently contained cells of abnormal karyotype in comparison to smaller tumors [26].

Guan et al. suggested that LC tumors were smaller in diameter compared to leiomyosarcoma (LMS) [27].

The analysis of the reviewed papers suggests that LC is not detected until histopathological examination of the postoperative material has been done.

To differentiate between LC and sarcoma stromale and leiomyosarcoma immunohistochemical methods must be applied, e.g. expression of desmin, h-caldesmon, CD10, and CD44V3 [28, 29].

The researchers believe that LC is not a transient form of leiomyosarcoma. Guan et al. examined 78 patients with LC over a longer period, and in 41 cases the tumor did not transform into malignancy [27].

However, some authors consider the nature of LC as "borderline" pathology and recommend longer clinical observation of those patients. Another fact, which has to be considered, is that some of the cases transformed into rare malignant histological subtypes of leiomyoma, e.g. lipoleiomyoma [30].

When LC was detected without concomitant malignancies in the genital organs, the patients did not require complementary treatment following the operation [27].

There were no postoperative complications observed in the cases quoted; the postoperative course of the patients operated on in our ward was uneventful, too.

\section{Conclusions}

The diagnosis of leiomyoma and LC of the fallopian tube, just like in other female genital organs, is not possible until microscopic histopathological examination has been done.

\section{Disclosure}

Author reports no conflict of interest.

\section{References}

1. Auguściak-Duma A, Sieroń AL. Molekularna charakterystyka guzów leiomyoma uteri na przykładzie wybranych składników macierzy pozakomórkowej. Postępy Hig Med Dośw 2008; 62: 148-165.

2. Madej P, Madej JA. Pathomorphosis of uterine myomas in women. Gin Pol Medproject 2008; 3: 32-42.

3. Sikora-Szczęśniak DL, Sikora W, Szczęśniak G. Leiomyoma cellulare in postoperative material: clinical cases. Med Stud 2013; 29: 144-151.

4. Ligon AH, Morton CC. Genetics of uterinae leiomyoma. Genes Chromosomes Cancer 2000; 28: 235-245.

5. Stewart EA, Morton CC. The genetics of uterine leiomyomata: what clinicians need to know. Obstet Gynecol 2006; 107: 917-921.

6. Darling TN, Pacheco-Rodriguez G, Gorio A, et al. Lymphangioleiomyomatosis and TSC2-/-cells. Lymphat Res Biol 2010; 8: 59-69.

7. Peng L, Wen $Y$, Han $Y$, et al. Expression of insulin-like growth factors (IGFs) and IGF signaling: molecular complexity in uterine leiomyomas. Fertil Steril 2009; 91: 2664-2675.

8. Peng Y, Laser J, Shi G, et al. Antiproliferative effects by Let-7 repression of high-mobility group A2 in uterine leiomyoma. Mol Cancer Res 2008; 6: 663-673.

9. Jakowicki JA. Onkologia ginekologiczna. Nowotwory macicy. In: Vademecum diagnostyki ginekologicznej. Jakowicki JA (ed.). Wydawnictwo BiFolium, Lublin 2009; 118-121.

10. Halder SK, Goodwin JS, Al-Hendy A. 1,25-Dihydroxyvitamin D3 reduces TGF-beta3-induced fibrosis-related gene expression in human uterine leiomyoma cells. J Clin Endocrinol Metab 2011; 96: E754-E762.

11. Fernandes $\mathrm{H}$, Naik CN, Swethadri GK, et al. Pure lipoma of the uterus: a rare case report. Indian J Pathol Microbiol 2007; 50: 800-801.

12. Taran FA, Weaver AL, Gostout BS, et al. Understanding cellular leiomyomas: a case-control study. Am J Obstet Gynecol 2010; 203: 109.e1-6.

13. Cisse $M$, Konate I, Dieng $M$, et al. Giant leiomyoma of fallopian tube: a rare aetiology of abdominal tumor. J Gynecol Obstet Biol Reprod 2008; 37: 799-801.

14. Mohapatra P, Sahoo S, Behera RN. Myoma of the fallopian tube: a rare case. J Evolution Medic Dent Scienc 2014; 3: 14418-14420.

15. Misao R, Niwa K, Iwagaki S, et al. Leiomyoma of the fallopian tube. Gynecol Obstet Invest 2000; 49: 279-280.

16. Yang CC, Wen KC, Chen P, et al. Primary leiomyoma of the fallopian tube: preoperative ultrasound findings. J Chin Med Assoc 2007; 70: 80-83.

17. Takongmo S, Nkwabong E, Sando Z, et al. A case report of a giant fallopian tube leiomyoma mimicking a mesenteric tumor. Clin Mother Child Health (AJOL) 2010; 7: 1229-1231.

18. Teymouri B, Sakhavar N, Mirteyomuri M. Leiomyoma of the fallopian tube: a case report and an unusual cause of abdominal pain. ZJRMS 2008; 10: 159-162.

19. Horisawa S, Ashida T, Sonoda M, et al. Torsion of fallopian tube leiomyoma treated by laparoscopically-assisted aproach: case report. Jap J Gynecol Obstet Endosc 2014; 30: 432-435.

20. Wen KC, Yang CC, Wang PH. Primary fallopian leiomyoma manager by laparoskopy. J Minim Invasive Gynecol 2005; 12: 193.

21. Ozkan Z, Gonen AN, Emir S, et al. Spontaneous ruprure of tubal leiomyoma causing haemoperitoneum. J Coll Physic Surg Pakistan 2014; 24 (Suppl 2): 91-92.

22. Mroueh J, Margono F, Feinkind L. Tubal pregnancy associated with ampullary tubal leiomyoma. Obstet Gynecol 1993; 81: 880-882.

23. Schust D, Stovall DW. Leiomyomas of the fallopian tube - a case report. J Reprod Med 1993; 39: 741-742.

24. Kwon GH, Rha SE, Ki EY, et al. Imaging findings of fallopian tube leiomyoma with myxoid degeneration: a case report. Clinical Imaging 2015; 39: 1105-1107.

25. Chmaj-Wierzchowska K, Buks J, Wierzchowski M, et al. Leiomyoma cellulare in the broad ligament of the uterus - case report and review of literature. Ginekol Pol 2012; 83: 301-304.

26. Lobel MK, Somasundaram P, Morton CC. The genetic heterogeneity of uterine leiomyomata. Obstet Gynecol Clin North Am 2006; 33: 13-39. 
27. Guan R, Zheng W, Xu M. A retrospective analysis of the clinicopayholog ic characteristics of uterine cellular leiomyomas in China Inter J Gynecol Obstet 2012; 118: 52-55.

28. Oliva E, Young RH, Amin MB, et al. An immunohistochemical analysis of endometrial stromal and smoth muscle tumors of the uterus: a study of 54 cases emphasizing the importance of using a panel because of overlap in immunoreactivity for individual antibodies. Am J Surg Pathol 2002; 26: 403-412.

29. Zhu XQ, Shi YF, Cheng XD, et al. Immunohistochemical markers in differential diagnosis of endometrial stromal sarcoma and cellular leiomyoma. Gynecol Oncol 2004; 92: 71-79.

30. McDonald AG, Dal Cin P, Ganguly A, et al. Liposarcoma arising in uterine lipoleiomyoma: a report of 3 cases and review of the literature. Am J Surg Pathol 2011; 35: 221-227. 